\title{
Photocounting distributions for exponentially decaying sources
}

\author{
Malvin Carl Teich and Howard C. Card \\ Columbia Rudiation Laboratory, Columbia University, New York, New York 10027
}

Received September 1, 1978; revised manuscript received February 1, 1979

\begin{abstract}
Exact photocounting distributions are obtained for a pulse of light whose intensity is exponentially decaying in time, when the underlying photon statistics are Poisson. It is assumed that the starting time for the sampling interval (which is of arbitrary duration) is uniformly distributed. The probability of registering $n$ counts in the fixed time $T$ is given in terms of the incomplete gamma function for $n \geq 1$ and in terms of the exponential integral for $n$ $=0$. Simple closed-form expressions are obtained for the count mean and variance. The results are expected to be of interest in certain studies involving spontaneous emission, radiation damage in solids, and nuclear counting. They will also be useful in neurobiology and psychophysics, since habituation and sensitization processes may sometimes be characterized by the same stochastic model.
\end{abstract}

In a variety of optical experiments, a pulse of light is generated in which the underlying Poisson photon statistics are modified by an intensity that decays exponentially in time. In some cases, as when the light is weak, it may be necessary or desirable to use photon-counting techniques to detect this signal.

In those situations in which the sampling interval (or time) $T$ is much greater than the decay time, essentially all of the light energy is contained in each sampling interval, so that the photocounting distribution will be Poisson of mean $\langle n\rangle=\eta \bar{I} T$. Here $\eta$ is the quantum efficiency of the detector, $\bar{I}$ is the time-averaged intensity of the light at the detector, and it is assumed that spatial effects can be ignored.

In this Letter, we derive the exact photocounting distribution expected when the sampling interval $T$ is not necessarily large in comparison with the decay time $\tau$ of the light. We also derive closed-form expressions for the count mean and variance. The results are valid for the repeated and exhaustive sampling of a single exponentially decaying light pulse, or for sampling from an ensemble of such pulses of identical height when the starting time of the sampling interval is uniformly distributed.

Figure 1 illustrates a decaying pulse of light and the times of interest in the present study. The quantity $\lambda(t)=\eta I(t)$ represents the effective intensity of the light, $\lambda_{0}$ is the peak value at $t=0$, and $\tau$ is the decay time (time required to reach $\lambda_{0} / e$ ). The starting time $t_{1}$ of the sampling interval (of length $T$ ) ranges between $t=$ 0 and $t=t_{2}$.

For a source of fixed intensity, the probability of registering $n$ counts in the time interval $T$, denoted by $p(n, T \mid W)$, is given by the simple Poisson distribution,

$$
p(n, T \mid W) \equiv p(n \mid W)=W^{n} e^{-W / n !},
$$

where $W$ represents the integrated effective intensity. When the intensity varies, Eq. (1) must be weighted by the values assumed by $W$. The result for the compound
Poisson distribution (doubly stochastic Poisson process) is $^{1-3}$

$p(n, T)=\langle p(n \mid W)\rangle_{W}=\int_{W}\left(W^{n} e^{-W / n !) P(W) \mathrm{d} W,}\right.$

where $P(W)$ is the probability density function for the integrated effective intensity $W$. With $\lambda(t)=$ $\lambda_{0} \exp (-t / \tau), W$ is

$$
\begin{aligned}
W\left(t_{1}, T\right) & =\int_{t_{1}}^{t_{1}+T} \lambda\left(t^{\prime}\right) \mathrm{d} t^{\prime} \\
& =\lambda_{0} \int_{t_{1}}^{t_{1}+T} \exp \left(-t^{\prime} / \tau\right) \mathrm{d} t^{\prime} \\
& =\lambda_{0} T\left[\frac{1-\exp (-T / \tau)}{T / \tau}\right] \exp \left(-t_{1} / \tau\right) \\
& =W_{0} \exp \left(-t_{1} / \tau\right),
\end{aligned}
$$

where $0 \leq t_{1} \leq t_{2}$ and $W_{0}$ depends on $T$ and $\tau$ but is independent of $t_{1}$. After briefly considering the simple case of a fixed starting time for the sampling interval, we consider the more interesting case in which the starting time $t_{1}$ is uniformly distributed.

In the case of fixed $t_{1}$, Eq. (3) reduces to

$$
W\left(t_{1}, T\right)=W_{0} \exp \left(-t_{1} / \tau\right)=W^{\prime},
$$

so that

$$
P(W)=\delta\left(W-W^{\prime}\right)
$$

and the overall counting distribution $p(n, T)$ remains a simple Poisson [Eq. (1)], but of mean $W^{\prime}$. Two special cases are of interest: (a) For $T / \tau \gg 1$,

$$
W^{\prime}=\lambda_{0} \tau \exp \left(-t_{1} / \tau\right),
$$

and (b) in the opposite limit, where $T / \tau \ll 1$,

$$
W^{\prime}=\lambda_{0} T \exp \left(-t_{1} / \tau\right) .
$$

These equations represent the mean count in a decay time [Eq. (6)] and in a sampling time [Eq. (7)], modified 


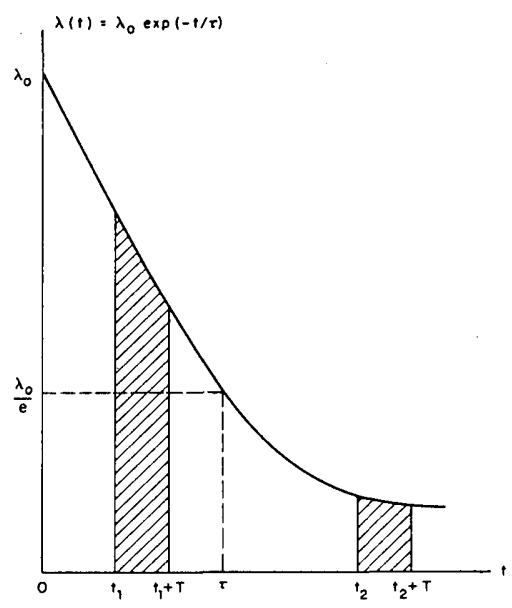

Fig. 1. Time course of the effective intensity (rate parameter) for an exponentially decaying source of decay time $\tau$ and initial value $\lambda_{0}$. The quantity $t_{1}$ represents the starting time of the counting interval, and $T$ is the duration of the counting interval. The starting time $t_{1}$ ranges between $t=0$ and $t=$ $t_{2}$.

by the exponential factor reflecting the loss of photons as the starting time for counting is delayed beyond the beginning of the light pulse.

The remainder of this Letter is concerned with the case in which the starting time $t_{1}$ has a uniform probability of being anywhere between $t=0$ and $t=t_{2}$. This gives rise to counting distributions that are not simple Poisson and corresponds to those physical situations of most interest to us in the present treatment. In this case,

$$
P(W)=p\left(t_{1}\right)\left|\mathrm{d} t_{1} / \mathrm{d} W\right| .
$$

From Eq. (3),

$$
\left(\mathrm{d} W / \mathrm{d} t_{1}\right)=-\left(W_{0} / \tau\right) \exp \left(-t_{1} / \tau\right)
$$

and, since $t_{1}$ is assumed to be uniformly distributed,

$$
p\left(t_{1}\right)=1 / t_{2}, \quad 0 \leq t_{1} \leq t_{2} .
$$

From Eq. (8), using Eqs. (9) and (10),

$$
\begin{array}{rlrl}
P(W) & =\left(\tau / t_{2}\right)\left[W_{0} \exp \left(-t_{1} / \tau\right)\right]^{-1} \\
& =\left(\frac{\tau}{t_{2}}\right) \frac{1}{W} & & 0 \leq t_{1} \leq t_{2} \\
& =0 & & \text { otherwise. }
\end{array}
$$

Specifying the region $0 \leq t_{1} \leq t_{2}$ in terms of $W$, we obtain the integrated effective intensity distribution

$$
\begin{array}{rlrl}
P(W) & =\left(\frac{\tau}{t_{2}}\right) \frac{1}{W} & W_{0} \exp \left(-t_{2} / \tau\right) \leq W \leq W_{0} \\
& =0 & & \text { otherwise, }
\end{array}
$$

as illustrated in Fig. 2. From Eq. (3),

$$
W_{0}=\lambda_{0} T\left[\frac{1-\exp (-T / \tau)}{T / \tau}\right] \text {. }
$$

It is easily verified that Eq. (12) is properly normalized.

The mean of $P(W)$, denoted by $\langle W\rangle$, is

$$
\begin{aligned}
\langle W\rangle & =\int_{W} W P(W) \mathrm{d} W \\
& =\frac{\tau}{t_{2}}\left[W_{0}-W_{0} \exp \left(-t_{2} / \tau\right)\right] \\
& =\lambda_{0} T\left[\frac{1-\exp (-T / \tau)}{T / \tau}\right]\left[\frac{1-\exp \left(-t_{2} / \tau\right)}{t_{2} / \tau}\right] \\
& =W_{0}\left(\tau / t_{2}\right)\left[1-\exp \left(-t_{2} / \tau\right)\right],
\end{aligned}
$$

which reduces in four special cases to

$$
\begin{aligned}
\langle W\rangle & =\left(\tau / t_{2}\right) \lambda_{0} \tau & & \text { for } t_{2} \gg \tau \text { and } T \gg \tau \\
& =\left(\tau / t_{2}\right) \lambda_{0} T & & \text { for } t_{2} \gg \tau \text { and } T \ll \tau \\
& =\lambda_{0} \tau & & \text { for } t_{2} \ll \tau \text { and } T \gg \tau \\
& =\lambda_{0} T & & \text { for } t_{2} \ll \tau \text { and } T \ll \tau .
\end{aligned}
$$

It is clear that when $T \ll \tau$, the relevant time parameter is $T$, whereas when $T \gg \tau$, it is $\tau$. Similarly, when $t_{2} \ll$ $\tau$, the relevant effective intensity (rate parameter) is the maximum value $\lambda_{0}$, whereas when $t_{2} \gg \tau$, it is the reduced value $\left(\tau / t_{2}\right) \lambda_{0}$. The variance of $P(W)$, denoted by $\left\langle(\Delta W)^{2}\right\rangle$, is

$$
\begin{aligned}
\left\langle(\Delta W)^{2}\right\rangle= & \left\langle W^{2}\right\rangle-\langle W\rangle^{2} \\
= & \left(\frac{\tau}{t_{2}}\right) W_{0}^{2}\left[\left(\frac{1}{2}-\frac{\tau}{t_{2}}\right)+2 \frac{\tau}{t_{2}} \exp \left(-t_{2} / \tau\right)\right. \\
& \left.\quad-\left(\frac{1}{2}+\frac{\tau}{t_{2}}\right) \exp \left(-2 t_{2} / \tau\right)\right] .
\end{aligned}
$$

In the count number $(n)$ domain, the mean $\langle n\rangle=$ $\eta\langle W\rangle=\langle W\rangle$ if a quantum efficiency $\eta$ of unity is assumed. ${ }^{1-3}$ Similarly, the variance is $\left\langle(\Delta n)^{2}\right\rangle=\eta\langle W\rangle$ $+\eta^{2}\left\langle(\Delta W)^{2}\right\rangle$ and, for $\eta=1$, the variance becomes $\langle W\rangle$ $+\left\langle(\Delta W)^{2}\right\rangle=\langle n\rangle+\left\langle(\Delta W)^{2}\right\rangle .1-3$

The exact counting distribution $p(n, T)$, from Eqs. (2) and (12), is expressed as

$$
\begin{array}{rlr}
p(n, T) & =\int_{W_{0 e^{-t 2 / \tau}}}^{W_{0}} \frac{W^{n} e^{-W}}{n !}\left(\frac{\tau}{t_{2}}\right) \frac{1}{W} \mathrm{~d} W \\
& =\left(\frac{\tau}{t_{2}}\right) \frac{1}{n !} \int_{W_{0} e^{-t 2 / \tau=a}}^{W_{0}=b} W^{n-1} e^{-W} \mathrm{~d} W & n \geq 1 \\
& =\left(\frac{\tau}{t_{2}}\right) \int_{W_{0} e^{-t_{2} / \tau=a}}^{W_{0}=b} \frac{1}{W} e^{-W} \mathrm{~d} W & n=0
\end{array}
$$

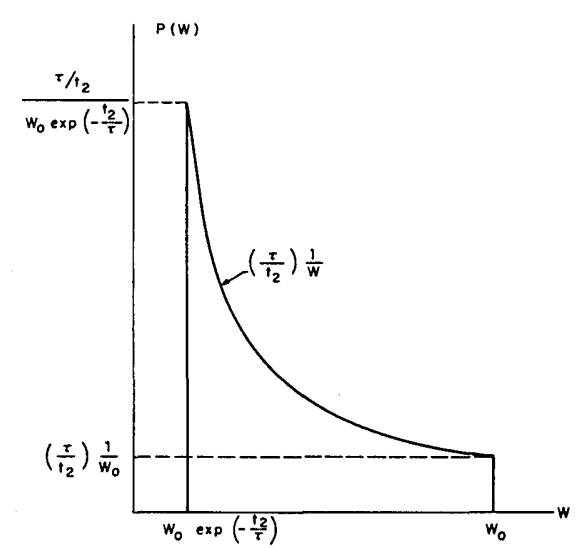

Fig. 2. Probability density function for the integrated effective intensity $W$. 
Since $\int_{a}^{b}=\int_{0}^{b}-\int_{0}^{a}$, and using Abramowitz and Stegun' ${ }^{4}$ definition for the incomplete gamma function

$$
P[n, x]=\frac{1}{\Gamma(n)} \int_{0}^{x} W^{n-1} e^{-W} \mathrm{~d} W
$$

with $\Gamma(n)=(n-1)$ ! for $n$ integer, we obtain

$$
p(n, T)=\left(\frac{\tau}{t_{2}}\right) \frac{1}{n}\left\{P\left[n, W_{0}\right]-P\left[n, W_{0} \exp \left(-t_{2} / \tau\right)\right]\right\}
$$

$$
n \geq 1 \text {. }
$$

Also, since $\int_{a}^{b}=\int_{a}^{\infty}-\int_{b}^{\infty}$, and using Abramowitz and Stegun's ${ }^{5}$ definition for the exponential integral

$$
E_{1}(x)=\int_{x}^{\infty} \frac{1}{W} e^{-W} \mathrm{~d} W,
$$

it also follows that

$$
p(0, T)=\left(\frac{\tau}{t_{2}}\right)\left\{E_{1}\left[W_{0} \exp \left(-t_{2} / \tau\right)\right]-E_{1}\left[W_{0}\right]\right\}
$$

where $W_{0}$ is given by Eq. (13).

The present results are expected to be of interest in the interpretation of photon-counting distributions generated by weak sources of phosphorescence ${ }^{6}$ and by other sources of spontaneous emission. The moments and photon-counting distribution presented in Eqs. (14), (16), (19), and (21) may permit a more accurate comparison of exponential decay theory with spontaneous-emission data. This could provide a determination of the extent to which the exponential spontaneous-emission law is violated, as a number of theoretical models suggest. ${ }^{7}$ One possible way in which such a spontaneous-emission experiment could be conducted is the following. A collection of atoms is prepared in the excited state, for example by irradiation with a brief but strong excitation pulse at time $t=0^{-}$. This has the effect of fixing the decaying effective intensity of all excited atoms at the value $\lambda_{0}$ at time $t=0$. The photon count $n$ from the sample during a fixed time interval $T$ is then recorded in the time slots $\left[t_{1}, t_{1}+T\right]$ with $0 \leq$ $t_{1} \leq t_{2}$, taking care to prepare the system again before each new interval is examined. ${ }^{7}$ This provides a uniform distribution of starting time on the interval $\left[0, t_{2}\right]$ and no restriction on $T$. A histogram of the relative frequencies of the counts is constructed, the experimental moments are calculated, and a comparison is effected with the theoretical results described above. We emphasize, however, that our calculations assume Poisson photon statistics for the underlying radiation. For certain sources, the appropriate underlying distribution will differ from Poisson. ${ }^{1-3,8,9}$ For chaotic light, the photon-counting distribution will often be closer to negative binomial ${ }^{8}$ than to Poisson; nevertheless, for situations in which the degeneracy parameter ${ }^{1,2} \delta \ll 1$, the underlying photon statistics will indeed be Poisson.

The analysis presented here also applies to various nuclear-counting experiments in which radioactive decay is monitored, since the underlying process is almost always Poisson and the mean count rate decreases exponentially in time in most cases. The model may also be used for certain problems encountered in lowlevel radiation damage in solids in which the counting takes place spatially rather than temporally. For ex- ample, a monochromatic $\mathrm{x}$-ray source will create damage that decays exponentially with perpendicular distance into the solid. The distribution of damage centers parallel to the surface is Poisson, so that our stochastic model describes the overall distribution of damage centers perpendicular to the surface.

In the distinctly different context of neurobiology, the amplitude of the postsynaptic potential (PSP) for certain neurons sometimes follows an approximately exponential time course during habituation and sensitization. This has been discussed by Castellucci et $a l .{ }^{10-12}$ for the gill-withdrawal reflex evoked by a weak tactile stimulation of the siphon skin in the marine mollusk Aplysia californica. Since the PSP is induced by the flow of discrete chemical neurotransmitter packets (quanta) across a synapse, and since the underlying statistics of the quanta are Poisson at sufficiently low arrival rates, ${ }^{13}$ the mathematical model we have developed should provide an appropriate description for quantal arrivals in the presence of habituation and sensitization. We would expect, furthermore, that habituation in neural counting and psychophysics may be similarly described.

This work was supported by the Joint Services Electronics Program (U.S. Army, U.S. Navy, and U.S. Air Force) under contract DAAG29-77-C-0019.

\section{References}

1. L. Mandel, "Fluctuations of photon beams: the distribution of the photo-electrons," Proc. Phys. Soc. (London) 74, 233-243 (1959).

2. J. Peřina, Coherence of Light (Van Nostrand Reinhold, London, 1972).

3. B. Saleh, Photoelectron Statistics (Springer-Verlag, Heidelberg, 1978).

4. M. Abramowitz and I. A. Stegun, eds., Handbook of Mathematical Functions with Formulas, Graphs, and Mathematical Tables [Nat. Bur. Stand. (U.S.), Appl. Math. Series 55, 1964/1972], p. 260, Eq. 6.5.1.

5. Ref. 4, p. 228, Eq. 5.1.1.

6. D. Curie, Luminescence in Crystals (Methuen, London, 1963).

7. P. L. Knight, "Non-Markovian effects in spontaneous emission: deviations from the exponential decay 'law'," in Coherence and Quantum Optics IV, L. Mandel and E. Wolf, eds. (Plenum, New York, 1978), pp. 635-645.

8. M. C. Teich, P. R. Prucnal, and G. Vannucci, "Optimum photon detection with a simple counting processor," Opt. Lett. 1, 208-210 (1977).

9. H. J. Kimble, M. Dagenais, and L. Mandel, "Photon antibunching in resonance fluorescence," Phys. Rev. Lett. 39, 691-695 (1977).

10. V. Castellucci, H. Pinsker, I. Kupfermann, and E. R. Kandel, "Neuronal mechanisms of habituation and dishabituation of the gill-withdrawal reflex in Aplysia," Science 167, 1745-1748 (1970).

11. V. Castellucci and E. R. Kandel, "A quantal analysis of the synaptic depression underlying habituation of the gill-withdrawal reflex in Aplysia," Proc. Nat. Acad. Sci. USA 71, 5004-5008 (1974).

12. J. H. Byrne, V. F. Castellucci, and E. R. Kandel, "Contribution of individual mechanoreceptor sensory neurons to defensive gill-withdrawal reflex in Aplysia," J. Neurophysiol. 41, 418-431 (1978).

13. J. del Castillo and B. Katz, "Quantal components of the end-plate potential," J. Physiol. (London) 124, 560-573 (1954). 